\title{
NOTE ON THE EQUIVALENCE OF NONSINGULAR PENCILS OF HERMITIAN MATRICES
}

\author{
JOHN WILLIAMSON
}

In a previous paper ${ }^{1}$ on the equivalence of nonsingular pencils of Hermitian matrices the restriction was made that the characteristic of the field in which the elements of the matrices lay be zero. It is the purpose of this note to show how this restriction can be removed and that all the results of this paper are true for fields of any characteristic different from two. Since many of the proofs involve division by the integer two, the case of characteristic two is not considered. To save unnecessary repetitions the notations used here conform with those of the previous paper.

If $A, B$ and $C, D$ are two pairs of Hermitian matrices, the pencil $A-\lambda B$ is said to be conjunctively equivalent to the pencil $C-\lambda D$ when there exists a nonsingular matrix $P$ such that $P^{*}(A-\lambda B) P$ $=C-\lambda D$, where $P^{*}$ is the conjugate transposed of $P$. If $B$ is nonsingular, the invariant factors of the pencil $A-\lambda B$ are the same as those of the pencil $A B^{-1}-\lambda E$. Let $M$ be any matrix similar to $A B^{-1}$ so that there exists a nonsingular matrix $P$ such that $P^{-1} A B^{-1} P=M$. Then

$$
P^{*} B^{-1}(A-\lambda B) B^{-1} P=P^{*} B^{-1} P(M-\lambda E)=R M-\lambda R .
$$

Therefore the pencil $A-\lambda B$ is conjunctively equivalent to the pencil $R M-\lambda R$ and, since $R M$ is Hermitian,

$$
R M=M^{*} R .
$$

Further, if $Q$ is any nonsingular matrix satisfying

$$
Q M=M^{*} Q,
$$

it follows from (1) that

$$
R=Q S,
$$

where $S$ is commutative with $M$. Moreover, if the pencil $R M-\lambda R$ is conjunctively equivalent to the pencil $G M-\lambda G$, so that there exists a nonsingular matrix $W$ such that $W^{*}(R M-\lambda R) W=G(M-\lambda E)$, the matrix $W$ is commutative with $M$.

In paper I the matrix $M$ is taken in the Wedderburn canonical

Received by the editors February 15, 1945.

${ }^{1} \mathrm{John}$ Williamson, The equivalence of non-singular pencils of Hermitian matrices in an arbitrary field, Amer. J. Math. vol. 57 (1935) pp. 475-490. This paper will be referred to as paper I. 
form $^{2}$ and a canonical form for the matrix $G$ is determined under a conjunctive transformation where the matrix $W$ of the transformation is commutative with $M$. Since this canonical form is valid in fields of characteristic different from zero, provided that $A B^{-1}-\lambda E$ has no elementary factor $[p(\lambda)]^{r}$ where $p(\lambda)$ is an inseparable polynomial, the only case not covered in paper $\mathrm{I}$ is that in which $A B^{-1}-\lambda E$ has one or more elementary factors of the type $[p(\lambda)]^{r}$ where $p(\lambda)$ is inseparable.

If, however, $M$ is taken to be the Jacobson canonical form ${ }^{3}$ no exceptions need be made. In fact with a slightly different interpretation of the symbols involved each proof in paper I holds word for word. The reason for this is that the forms of the matrix $Q$, satisfying (2), and of the matrices $S$ and $W$ commutative with $M$ are essentially the same as before.

Let $[p(\lambda)]^{r}$ be an elementary factor of the pencil $A-\lambda B$ where $p(\lambda)=\lambda^{n}-a_{n} \lambda^{n-1}-a_{n-1} \lambda^{n-2}-\cdots-a_{1}$, let

$$
p=\left(\begin{array}{ccccc}
0 & 1 & 0 & \cdots & 0 \\
0 & 0 & 1 & \cdots & 0 \\
. & . & . & \cdots & . \\
0 & 0 & 0 & \cdots & 1 \\
a_{1} & a_{2} & a_{3} & \cdots & a_{n}
\end{array}\right)
$$

be the companion matrix of $p(\lambda)$ and let

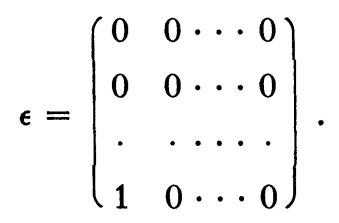

If $M$ is the Jacobson canonical form of $A B^{-1}, M$ is a diagonal block matrix with one block the $r n$-rowed square matrix

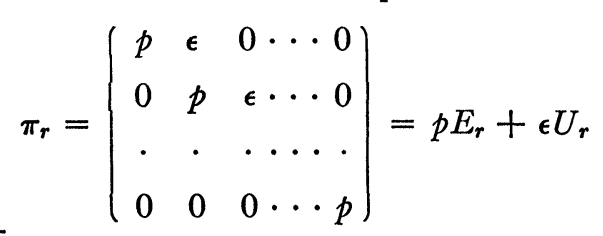

${ }^{2}$ J. H. M. Wedderburn, Lectures on matrices, Amer. Math. Soc. Colloquium Publications, vol. 17, New York, 1934, pp. 123-125.

3 J. H. M. Wedderburn, The canonical form of a matrix, Ann. of Math. vol. 39 (1938) pp. 178-180. C. C. MacDuffee, Vectors and matrices, Carus Mathematical Monographs, 1943, p. 136. 
corresponding to the elementary factor $[p(\lambda)]^{r}$. Any matrix commutative with $\pi_{r}$ is a polynomial $f\left(\pi_{r}\right)$ in $\pi_{r}$. Since the minimal polynomial of $\pi_{r}$ is $[p(x)]^{r}$ of degree $n r$,

$$
f\left(\pi_{r}\right)=f_{0}\left(\pi_{r}\right)+f_{1}\left(\pi_{r}\right) p\left(\pi_{r}\right)+\cdots+f_{r-1}\left(\pi_{r}\right)\left\lfloor p\left(\pi_{r}\right)\right]^{r-1}
$$

where $f_{i}\left(\pi_{r}\right)$ is a polynomial in $\pi_{r}$ of degree not greater than $n-1$, $i=0,1, \cdots, r-1$.

A routine but long calculation shows that $p\left(\pi_{r}\right)=e U_{r}$ where $e$ is the unit matrix of order $n$. It is however not really necessary to make this calculation. The coefficient of $U_{r}$ in $\pi_{r}^{t+1}$ is $w_{t+1}=\sum_{j=0}^{t} p^{i} \epsilon p^{t-i}$ and therefore $p w_{t+1}-w_{t+1} p=p^{t+1} \epsilon-\epsilon p^{t+1}$. Consequently, if $h$ is the coefficient of $U_{r}$ in $p\left(\pi_{r}\right), p h-h p=p(p) \epsilon-\epsilon p(p)=0$ so that $h$ is commutative with $p$. Since $h$ is not zero, $h$ is nonsingular and, if, in $\pi_{r}$, $\epsilon$ is replaced by $h^{-1} \epsilon, p\left(\pi_{r}\right)=h^{-1} h U_{r}=e U_{r}$.

Accordingly (4) assumes the form

$$
f\left(\pi_{r}\right)=f_{0}\left(\pi_{r}\right)+f_{1}\left(\pi_{r}\right) U_{r}+\cdots+f_{r-1}\left(\pi_{r}\right) U_{r}^{r-1} .
$$

If $p(\lambda)$ is separable and $M$ is the Wedderburn canonical form, in paper I $N_{r}=p E_{r}+e U_{r}$ takes the place of $\pi_{r}$. Any matrix commutative with $N_{r}$ is of the form

$$
f\left(N_{r}\right)=f_{0}(p) E_{r}+f_{1}(p) U_{r}+\cdots+f_{r-1}(p) U_{r}^{r-1} .
$$

It is obvious that (5) can be obtained from (6) by replacing $p$ by $\pi_{r}$. Further, if

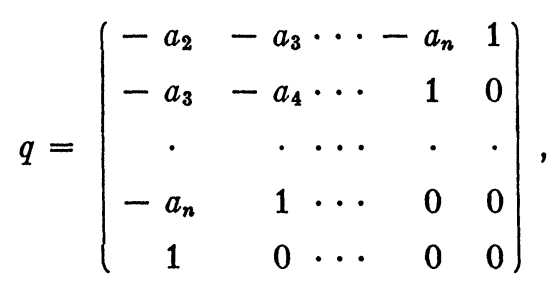

$q=q^{\prime}, q p=p^{\prime} q$ and $q \epsilon=\epsilon^{\prime} q$. Accordingly the matrix

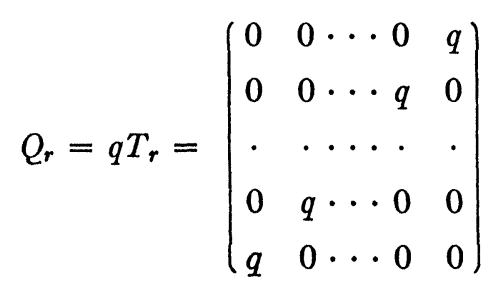

satisfies the equation $Q_{r} \pi_{r}=\pi_{r}^{\prime} Q_{r}$. Apart from the definition of $q$, the matrix $Q_{r}$ is the same as the corresponding matrix in paper I (see formula (11)). 
In the proofs of paper I the only use made of the fact that the elements of the matrices under consideration are polynomials in $p$ is the following. If $g(x)$ is a polynomial of degree not greater than $n-1$ and if the matrix $g(p)$ is singular, then $g(p)$ is zero. However the same is true if $p$ is replaced by $\pi_{r}$, for $\left|g\left(\pi_{r}\right)\right|=|g(p)| r$. Accordingly, if $p$ is replaced by $\pi_{r}$ and $q$ by the matrix defined in (7), the proofs and results of paper I are valid for fields of any characteristic different from two.

Queens College 